\title{
High-Grade Bursal Side Rotator-Cuff Repair: A Surgical Outcome Review
}

\author{
Renaldi Prasetia' \\ Erica Kholinne ${ }^{2}$ \\ Prettysia Suvarly ${ }^{3}$ \\ Wendy Yolanda Rosa' \\ Yuni Susanti Pratiwi ${ }^{4,5}$ \\ Herry Herman' \\ Hermawan Nagar Rasyid' \\ Bancha Chernchujit ${ }^{6}$ \\ Ronny Lesmana ${ }^{4,5}$ \\ 'Department of Orthopaedics - \\ Traumatology, University Padjadjaran, \\ Hasan-Sadikin General Hospital, Bandung, \\ Indonesia; ${ }^{2}$ Department of Orthopaedic \\ Surgery, Trisakti University, St Carolus \\ Hospital, Jakarta, Indonesia; ${ }^{3}$ Department \\ of Orthopaedics and Traumatology, Pelita \\ Harapan University, Siloam Hospitals \\ Lippo Village, Tangerang, Indonesia; \\ ${ }^{4}$ Department of Anatomy, Physiology and \\ Biology Cell, Universitas Padjadjaran, \\ Bandung, Indonesia; ${ }^{5}$ Central Laboratory, \\ Universitas Padjadjaran, Bandung, \\ Indonesia; ${ }^{6}$ Department of Orthopaedics, \\ Thammasat University Hospital, Pathum \\ Thani, Thailand
}

Purpose: We aimed to evaluate surgical outcomes of high-grade bursal rotator cuff-tear repairs.

Methods: This systematic review was performed in May 2020 in accordance with the Preferred Reporting Items for Systematic Reviews and Meta-Analyses guidelines using PubMed. Inclusion criteria were English-language studies reporting the results of pain improvement, functional outcome scores, and radiographic examinations after repair of bursal side partial rotator-cuff tears at any time point in patients of any age and with all levels of evidence. Exclusion criteria were articles not in English, in vitro or animal studies, epidemiological studies, and such article types as technical notes or narrative reviews.

Results: Of 58 articles, five were included in this study, of which three and two had level III and IV evidence, respectively, four were comparative studies, and one was a case series. Visual analogue scales were used in four of the five studies, all showing improvement in pain assessment from 5.87 preoperatively to 1.02 postoperatively. All five studies showed significant improvement on each functional outcome score at the final follow-up. The retear rate for all studies was $10.97 \%$ (27 of 246).

Conclusion: High-grade bursal side partial-thickness rotator cuff-tear repair gave satisfactory results in terms of pain scores, range of motion, and functional outcomes. The retear rate was still considerably high (10.9\%), necessitating better understanding of the basic science, such as molecular mechanisms during adaptation, to improve the surgical technique.

Keywords: partial rotator-cuff tear, remnant preservation, bursal takedown

\section{Introduction}

Partial-thickness rotator cuff tears (PTRCTs) are often encountered in common orthopedic practice. Ellman arthroscopically classified PTRCTs according to the location (articular, bursal, or interstitial) and depth of the tear. ${ }^{1}$ Ellman grade III tears, involving $>6 \mathrm{~mm}$ or $50 \%$ of tendon thickness, are also known as high-grade PTRCTs. $^{2}$

The usual first-line treatment of patients with PTRCT symptoms is a trial of at least 3-6 months of nonoperative treatment, which includes modification of activity and use of analgesic and anti-inflammatory drugs. Corticosteroid injections are an adjunct to further nonoperative treatment, and are administered in the subacromial space in the case of bursal lesions. Bursal side tears usually occur in middle-aged or elderly ( $>40$ years) patients and are less common than articular side tears. ${ }^{3,4}$ Patients with symptoms of pain and disability that persist after adequate trials of nonoperative treatment should be considered for surgery. Patients with a bursal side tear should be closely monitored for their response to nonoperative management,
Correspondence: Renaldi Prasetia Department of Orthopaedics Traumatology, University Padjadjaran, Hasan-Sadikin General Hospital, Bandung 40I6I, Indonesia

Email renaldi@unpad.ac.id 
with consideration given to those with tears $>50 \%$ of the thickness for early surgical intervention. ${ }^{5}$ Tear size and depth, patient age and activity level, and cause of the tear also have to be considered. ${ }^{6,7}$ The recommended treatment for bursal side tears includes debridement with or without acromioplasty for grade I tears (low grade) and rotator cuff-tendon repair for grade II and III tears (high grade). ${ }^{8,9}$ For grade III articular tears and grade II or III bursal tears, tendon repair should be performed using a technique based on the surgeon's preference and the patient's goals. To date, there is still no consensus regarding surgical management for high-grade bursal side RCT. The debate remains between preserving the remnant fibers of the injured tendon as much as possible and debriding the tissue ("takedown") by conversion to a full-thickness tear. The former has the downside of not having an optimal visual field, but with the consideration of respecting the remnant tissue. Furthermore, in a retracted bursal side $\mathrm{RCT}$, a simple repair might be insufficient, because the repaired tendon could remain as an intratendinous tear of the rotator cuff. ${ }^{10}$ The latter is usually chosen if the tear is nearly full or if the remaining articular fibers are of poor quality. $^{6}$

From the various studies that have been conducted, though the results of various surgical procedures in the management of high-grade bursal side RCTs have shown satisfactory results, there is no definite agreement on the best management procedure that can be used as a reference. The current systematic review aimed to evaluate the surgical outcomes of high-grade bursal RCT repair.

\section{Methods}

\section{Literature Search}

This systematic review was done according to the Preferred Reporting Items for Systematic Reviews and Meta-Analyses guidelines, ${ }^{11}$ used the PubMed database, and was performed in May 2020 (Figure 1). The search string used was [(partial rotator cuff tear OR partial thickness rotator cuff tear) AND (clinical outcome) AND (repair integrity)]. No limits were placed on the date of publication. Inclusion criteria were English-language studies reporting the results of pain improvement, functional outcome scores, and radiographic examinations after repair of bursal side partial RCT at any time point in patients of any age and all levels of evidence. Exclusion criteria were as follows: studies not in English, in vitro or animal studies, epidemiological studies, and such articles types as technical notes or narrative reviews.

Two reviewers independently screened all articles for relevance based on titles and abstracts according to the inclusion and exclusion criteria. We also conducted citation tracking from the bibliographies of the retrieved studies to locate additional related articles. The same reviewers obtained and reviewed the full text of all articles not excluded during the initial screening process for possible inclusion in the systematic review. Any disagreement on article eligibility was resolved through discussion between two orthopedic surgeons.

\section{Data Extraction}

Patient demographics, study characteristics, surgical technique, outcome scores, and structural integrity after the operation were abstracted from each study that met the inclusion criteria. Data were extracted by a reviewer and verified by another reviewer. Two orthopedic surgeons resolved any disagreement through discussion. Study characteristics included author names, year of publication, study design, number of patients, and length of followup. Patient demographic characteristics included sex, age, and comorbidity (if any). Outcomes of interest included preoperative and postoperative pain improvement, preoperative and postoperative functional outcome scores, preoperative and postoperative range of movement, and postoperative structural integrity using magnetic resonance imaging (MRI), ultrasound (US), or other imaging modalities. Descriptive statistics were used to report study and demographic characteristics and preoperative and postoperative outcomes of all patients. Given the heterogeneity between bursal takedown (BTD) and remnant preservation (RP) for the surgical procedure, we decided to extract data on and descriptive comparisons of pain improvement, functional outcome improvement, and healing status or retear rate between BTD and RP groups. Complications of the procedure were defined as retear rate. Meta-analysis and quantitative comparison across studies were considered inappropriate, because of the heterogeneity in surgical technique, functional outcome scores, and insufficient data from several publications (eg, standard deviations and the minimum and maximum scores).

\section{Quality Assessment}

The methodological quality of the studies was analyzed using the MINORS criteria. This score was used to qualify the 


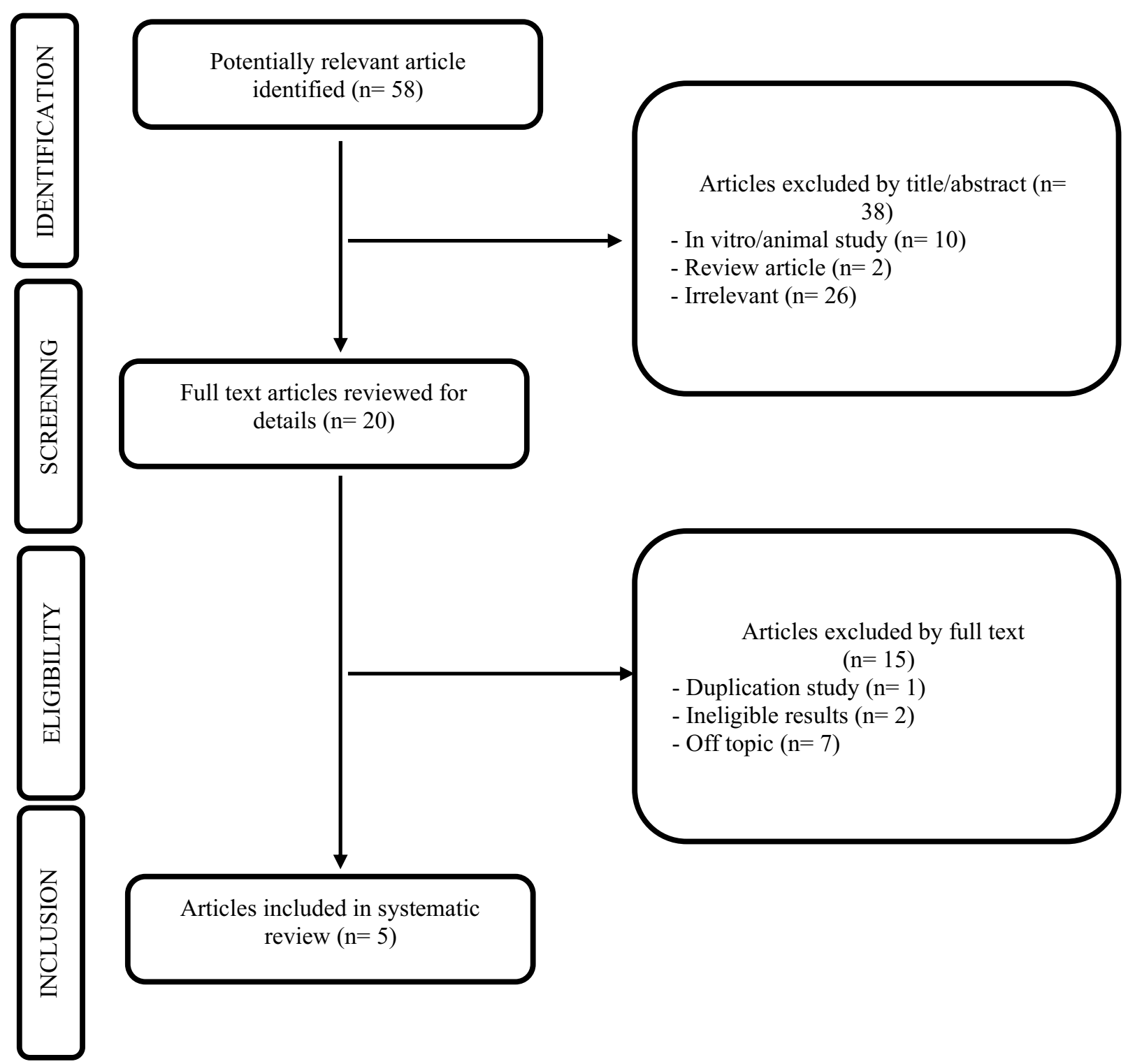

Figure I Flowchart of search strategy according to the Preferred Reporting Items for Systematic Reviews Aand Meta-analysis guidelines.

assessment of nonrandomized studies using seven specific criteria for noncomparative studies and an additional five for comparative studies. A high total score means that the article had a low risk of bias. In the current study, a score $>60 \%$ of the total score with the MINORS evaluation tool (14 of 24 points or 9 of 16 points) was considered high quality. All studies included were also assessed for level of evidence according to the Oxford Centre for Evidence-Based Medicine.

\section{Results}

\section{Literature Search}

The search results are summarized in Figure 1. Of the 58 articles, five were included in this study, of which three and two had level III and IV evidence, respectively, four were comparative studies, and one was a case series (Table 1). From these studies, only patients with high-grade bursal side PTRCTs who had undergone operations were included. Four of the five articles were used in this study for comparison between the BTD and RP groups.

\section{Study Quality}

The mean MINORS score was $12.4 \pm 2.4$. The corresponding values for each study shown in Table 1 .

\section{Patient Characteristics}

In total, 258 patients were assessed, with a mean 51.6 patients per study. There were 131 male patients 
Table I General Characteristics of Included Studies

\begin{tabular}{|l|c|c|c|c|c|c|}
\hline & Year & Study Design & $\begin{array}{c}\text { Level of } \\
\text { EBM }\end{array}$ & $\begin{array}{c}\text { MINORS } \\
\text { Score }\end{array}$ & $\begin{array}{c}\text { Functional Scores } \\
\text { Radiographic } \\
\text { Evaluation }\end{array}$ \\
\hline${\text { Kim et } \text { al }^{7}}$ & 2013 & Prospective Cohort study & III & II & ASES, UCLA, and Constant & MRI or US \\
Xiao and Cuil $^{16}$ & 2015 & Retrospective case study & IV & 9 & UCLA and Constant & MRI \\
Shin et al $^{21}$ & 2015 & Retrospective cohort study & III & 15 & ASES and Constant score & MRI \\
Kim et al $^{10}$ & 2013 & Case control study & III & 13 & ASES, SST, and UCLA shoulder & MRA \\
Koh et al $^{15}$ & 2011 & Case series & IV & I4 & ASES and Constant & MRI \\
\hline
\end{tabular}

Abbreviations: EBM, evidence-based medicine; ASES, American Shoulder and Elbow Surgeons; UCLA, University of California Los Angeles; SST, Simple Shoulder Test; MRI, magnetic resonance imaging; US, ultrasound; MRA, magnetic resonance arthrography.

$(50.73 \%)$ and 127 female patients (49.22\%). Mean age 48.9-58.7 years, with a mean age across all studies of 54.5 years.

\section{Surgical Technique and Rehabilitation}

Of the five studies, three studies reported on surgical procedures in the beach-chair position and the remaining two studies in the lateral decubitus position. One study reported using standard posterior and anterior portals, and one used the posterolateral portal as the viewing portal and the anterolateral or anterior portal as the working portal. One study reported acromioplasty in all patients, and the remaining four studies acromioplasty limited to the impinged area, removal of osteophytes, and when the acromion had a hooked shape. Subacromial decompression was performed in two studies, one study in all patients, the other one only in 21 patients with evidence of subacromial impingement. For the surgical procedure, one study reported BTD, two studies RP, and two studies BTD and RP. Two studies reported using the double-row technique, one only the single-row technique, one the double-row technique in 29 patients and single-row technique in 30 , and one study the double-row technique in 37 patients and single-row technique in 47 patients. Four studies reported full-thickness conversion: two studies in all patients, one only in 37 patients, and one limited to where the tear involved $>50 \%$ thickness of the tendon.

For rehabilitation, the arm was immobilized using a sling with an abduction pillow (three studies) and shoulder brace (two studies). Gentle pendulum nd passive range-of-motion exercises were performed on the first day after surgery in one study, third day after surgery in one study, and first week after surgery in one study, and started from 2 weeks after surgery in one study and 3-6 weeks after surgery in one study. Active range-of-motion exercise was performed from 6 weeks after surgery in four studies and from 12 weeks after surgery in one study. All studies reported starting strengthening exercises from 3 months after surgery and gradually returning to sports from 6 months after surgery as tolerated.

\section{Pain}

A visual analogue scale (VAS) was used in four of the five studies, all showing improvement in pain assessment from 5.87 preoperatively to 1.02 postoperatively. VAS scores between the BTD and RP groups also improved postoperatively (BTD 5.9 to 1 , RP 5.3 to 1.2 ).

Corresponding values for each study are summarized in Table 2 for all patients and Table 3 for comparison between BTD versus RP groups.

\section{Functional Outcomes}

Four measures were used to assess functional outcomes: American Shoulder and Elbow Surgeons Shoulder (ASES) score (four studies), constant score (four studies), University of California, Los Angeles (UCLA) shoulder score (three studies), and Simple Shoulder Test (SST) score (one study). All five studies showed significant improvement on each functional outcome score at the final follow-up. The most commonly used measures were the constant score $(79.1 \%$ of patients) and ASES score (77.1\% of patients), followed by the UCLA score $(52.7 \%$ of patients) and SST score (20.9\% of patients). Mean constant score improved from 63.3 preoperatively to 83.55 postoperatively (BTD 69.6-86.15, RP 64.4-89.6). TMean ASES score improved from 45.5 preoperatively to 90.0 postoperatively (BTD 49.2-92.4, RP 43.5-87.9). Mean UCLA score improved from 16.7 preoperatively to 32.2 postoperatively (BTD 19.8-32.5, RP 17.3-33.4). Mean SST score improved from 4.7 preoperatively to 10.0 postoperatively. Corresponding values for each study are presnted in Table 3 . 

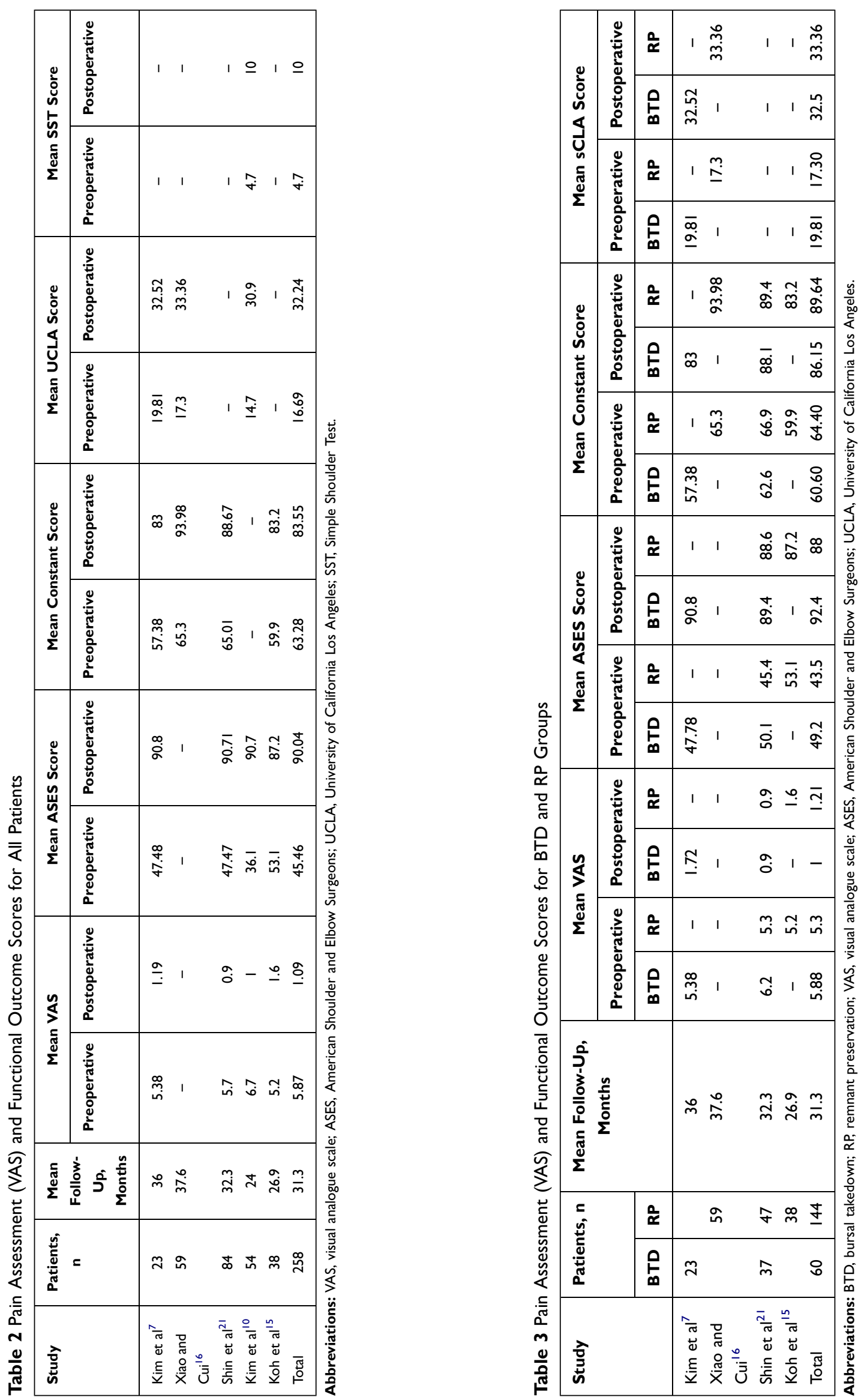


\section{Range of Movement}

Two studies reported improvement in mean forward flexion - from 148.87 preoperatively to 178.75 postoperatively (BTD 148.57-178.1, RP 148-179) — and improvement in mean abduction: from 134.91 preoperatively to 169.8 postoperatively (BTD 119.29-169.3, RP 141-170). Only one study reported mean internal rotation and external rotation scores. Internal rotation also improved from L5 preoperatively to T12 postoperatively. External rotation improved from 41 preoperatively to 44 postoperatively. However, because of insufficient data on internal and external rotation from the study that used RP for the surgical technique, the mean internal and external rotation could not be compared between the BTD and RP groups. The corresponding values for each study are summarized in Table 4.

\section{Retear Rates}

Five studies reported retear rates at the final follow-up using MRI, magnetic resonance arthrography, or US. The retear rate from all studies was $10.97 \%$ (27 of 246), and that in the BTD and RP groups $8.6 \%$ (five of 58) and $11.94 \%$ (16 of 134), respectively. The healing state in each study is summarized in Table 5, and the comparison of retear rates between the BTD and RP groups summarized in Table 6.

\section{Discussion}

The most important finding of this systematic review was that the surgical treatment of high-grade bursal side RCT shows favorable results. We included both clinical and radiological assessments of high-grade bursal side rotatorcuff repair.

Standard bursal side PTRCT surgical treatment remains controversial. ${ }^{3}$ Some of the literature supports full-thickness conversion repair. ${ }^{12,13}$ Other studies have found that for PTRCTs, intact articular side rotator cuff-fiber preservation can be done. ${ }^{6,7,10,14-19}$ There were five studes that mentioned repair integrity and clinical outcomes. ${ }^{7,12,13,15,16}$ Only several study that confirm the PTRCTs in high-grade settings ( $>50 \%$ partial tear). One study preserved any intact articular fibers while removing diseased tissue after bursectomy and debriding diseased tissue on the bursal side. ${ }^{20}$ There are three possible reasons to preserve the articular footprint in repairing a bursal side RCT. The first is to protect the bursal side repair (intact articular fibers act as an internal splint). Synovial fluid is a substance that can disrupt tissue healing. The second is to recreate a wide and anatomic footprint from

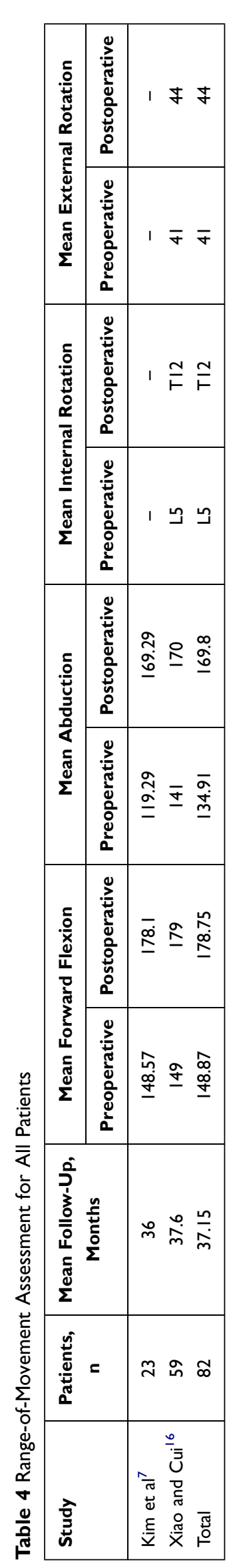


Table 5 Healing Status

\begin{tabular}{|l|c|c|c|l|}
\hline Study & Method & $\begin{array}{c}\text { Patients, } \\
\mathbf{n}\end{array}$ & $\begin{array}{c}\text { Mean } \\
\text { Follow-Up, } \\
\text { Months }\end{array}$ & \multicolumn{1}{|c|}{ Healing } \\
\hline Kim et al $^{7}$ & $\begin{array}{c}\text { MRI or } \\
\text { US }\end{array}$ & 21 & 36 & $\begin{array}{l}\text { Complete: } 19 \\
\text { Retear: } 2\end{array}$ \\
\hline $\begin{array}{l}\text { Xiao and } \\
\text { Cui }\end{array}$ & MRI & 49 & 37.6 & $\begin{array}{l}\text { Complete: } 41 \\
\text { Retear: } 8\end{array}$ \\
\hline Shin et al & MRI & 84 & 32.3 & $\begin{array}{l}\text { Complete: } 77 \\
\text { Retear: } 7\end{array}$ \\
\hline Kim et al & MRA & 54 & 24 & $\begin{array}{l}\text { Complete: } 48 \\
\text { Retear: } 6\end{array}$ \\
\hline Koh et al & MRI & 38 & 26.9 & $\begin{array}{l}\text { Complete: } 34 \\
\text { Retear: } 4\end{array}$ \\
\hline Total & - & 246 & 31.3 & $\begin{array}{l}\text { Complete: } \\
219 \text { (89.03\%) } \\
\text { Retear: } 27 \\
\text { (I0.97\%) }\end{array}$ \\
\hline
\end{tabular}

Table 6 Retear Rate in BTD Group vs RP Group

\begin{tabular}{|l|c|c|c|c|}
\hline \multirow{2}{*}{ Study } & \multicolumn{2}{|c|}{ Retear Rate } & \multicolumn{2}{c|}{ Patients, n } \\
\cline { 2 - 5 } & BTD & RP & BTD & RP \\
\hline Kim et al $^{7}$ & $9.52 \%(2 / 21)$ & & 21 & \\
Xiao and Cui $^{16}$ & & $16.33 \%(8 / 49)$ & & 49 \\
Shin et al & & $8.5 \%(4 / 47)$ & 37 & 47 \\
Koh et al & $8.1 \%(3 / 37)$ & $10.53 \%(4 / 38)$ & & 38 \\
Total & & $11.94 \%(16 / 134)$ & 58 & 134 \\
\hline
\end{tabular}

Abbreviations: BTD, bursal takedown RP, remnant preservation.

the intact articular attachment after the repair procedure. The third is to prevent overlateralization of the footprint with respect to the native length tension that may reduce the risk of having a retear. ${ }^{14}$

Anderson et al reported outcomes of patients who had had high-grade bursal side RCT arthroscopic repairs: 27 patients with an intact articular surface but a high-grade bursal side tear with $>7 \mathrm{~mm}$ exposed tuberosity were treated with subacromial decompression and arthroscopic rotator-cuff repair. Serial examination and the L'Insalata Shoulder Rating Questionnaire to assess outcomes showed that arthroscopic repair of high-grade bursal side RCTs resulted in a high degree of functional improvement, low surgical morbidity, and high patient satisfaction. These outcomes might be favorable to previous arthroscopic decompression and/ or debridement reports. ${ }^{22}$
Interestingly, we observed that retears were the most common complication following rotator-cuff repair $10.97 \%$ ( 27 of 246 patients) in the five studies. A retear following rotator-cuff repair does not always imply failure of healing. The retear rate was greater in the RP group than the BTD group. Various factors have been shown to influence failure of healing, including the technique used. Theoretically, this can be secondary to early mechanical failure because of unsound repair or biological healing failure, regardless of good repair or a recurrent tear after good healing from the initial tear. From the five studies, the retear rate of RCTs with the BTD procedure was better than that of the RP technique. We postulated whether the tissue retained in the surgical procedures was pathological tissue of the remaining rotator cuff, which may have caused recurrent pain. There have been no studies investigating whether remnant tissue is pathological or physiological tissue that can be preserved. ${ }^{23,24}$

The limitations of this study include the modest number of articles due to the narrow inclusion criteria, which may limit generalization to the wider population. The outcome-measurement tools were heterogeneously reported, preventing direct comparison of outcomes and undermining the need for standardized methods. Also, data were collected retrospectively without a control group. However, the studies were classified as high quality based on MINORS criteria.

\section{Conclusion}

High-grade bursal side PTRCT repair with RP or BTD technique had satisfactory results in terms of VAS, ROM, and functional outcome scores. The retear rate was higher with RP than BTD, pointing to the need for further understanding of the basic science of remnant rotator-cuff tissue to improve the outcome of rotator cuff-repair surgery.

\section{Acknowledgments}

The study was supported by a Review Article Writing Grant Universitas Padjadjaran to YSP (1733/UN6.3.1/LT/ 2020) and underwent grammar correction by Enago.

\section{Disclosure}

The authors report no conflicts of interest in this work.

\section{References}

1. Ellman H. Diagnosis and treatment of incomplete rotator cuff tears. Clin Orthop Relat Res. 1990;254:64-74. 
2. Sambandam SN, Khanna V, Gul A, Mounasamy V. Rotator cuff tears: an evidence based approach. World J Orthop. 2015;6(11):902-918. doi:10.5312/wjo.v6.i11.902

3. Papalia R, Franceschi F, Del Buono A, Maffulli N. Results of surgical management of symptomatic shoulders with partial thickness tears of the rotator cuff. Br Med Bull. 2011;141-154. doi:10.1093/bmb/ldq040

4. Aydin N, Karaismailoglu B. High-grade bursal-side partial rotator cuff tears: comparison of mid- and long-term results following arthroscopic repair after conversion to a full-thickness tear. J Orthop Surg Res. 2017;12(1):1-6.

5. Borbas P. Surgical treatment options for partial rotator cuff tears: a review of the literature. Int $J$ Orthop. 2019. doi:10.17554/j. issn.2311-5106.2019.06.281-6

6. Yoo JC, Ahn JH, Lee SH, Kim JH. Arthroscopic full-layer repair of bursal-side partial-thickness rotator cuff tears: a small-window technique. J Arthrosc Relat Surg. 2007;23(8):1-4. doi:10.1016/j. arthro.2006.11.008

7. Kim Y-S, Lee H-J, Bae S-H, JunJin H, Song HS. Outcome comparison between in situ repair versus tear completion repair for partial thickness rotator cuff tears. Arthrosc J Arthrosc Relat Surg. 2015;31 (11):2191-2198. doi:10.1016/j.arthro.2015.05.016

8. Mathiasen R, Hogrefe C. Evaluation and management of rotator cuff tears: a primary care perspective. Curr Rev Musculoskelet Med. 2018;11(1):72-76. doi:10.1007/s12178-018-9471-6

9. Fukuda $H$. The management of partial-thickness tears of the rotator cuff. J Bone Jt Surg Ser B. 2003;85(1):3-11. doi:10.1302/0301620X.85B1.13846

10. Kim K, Rhee K, Kim HS, Kim P. Arthroscopic footprint reconstruction of bursal-side delaminated rotator cuff tears using the suture-bridge technique. Knee Surg Sport Traumatol Arthrosc. 2009;17(7):840-843. doi:10.1007/s00167-009-0730-3

11. Moher D, Liberati A, Tetzlaff J, Altman DG. Preferred reporting items for systematic reviews and meta-analyses: the PRISMA statement. Ann Intern Med. 2009;151(4):264-9, W64. doi:10.7326/ 0003-4819-151-4-200908180-00135

12. Kamath G, Galatz LM, Keener JD, Teefey S, Middleton W, Yamaguchi K. Tendon integrity and functional outcome after arthroscopic repair of high-grade partial-thickness supraspinatus tears. J Bone Jt Surg Am. 2009;91:1055-1062. doi:10.2106/JBJS.G.00118

13. Kim KC, Shin HD, Cha SM, Park JY. Repair integrity and functional outcome after arthroscopic conversion to a full-thickness rotator cuff. Am J Sports Med. 2014;20:10.
14. Wolff AB, Magit DP, Miller SR, Wyman J, Sethi PM. Arthroscopic fixation of bursal-sided rotator cuff tears. J Arthrosc Relat Surg. 2006;22(11):1-4.

15. Koh KH, Shon MS, Lim TK, Yoo JC. Clinical and magnetic resonance imaging results of arthroscopic full-layer repair of bursal-side partial-thickness rotator cuff tears. Am J Sports Med. 2008;39(8):1660-1667. doi:10.1177/036354651141 2165

16. Xiao J, Cui G. Clinical and structural results of arthroscopic repair of bursal-side partial-thickness rotator cuff tears. J Shoulder Elb Surg. 2015;24(2):e41-6. doi:10.1016/j.jse.2014.07.008

17. Cheon K, Kwang K, Rhee J. Mattress suture-bridge technique for bursal-side partial-thickness rotator cuff tears. Arch Orthop Trauma Surg. 2010;130:407-411. doi:10.1007/s00402009-0996-4

18. Chernchujit B, Hamid MAS, Aimprasittichai S. Knotless suture bridge technique in high-grade bursal-sided rotator cuff tears. is this the way forward? Arthrosc Tech. 2017;6(6):e2259-63.

19. Shin S, Kook S, Rao N, Seo M. Clinical outcomes of modified Mason-Allen single-row repair for bursal-sided partial-thickness rotator cuff tears. Am J Sports Med. 2015;20:10.

20. Prasetia R, Sukhapradit B, Chernchujit B. Clinical features and repair integrity after knotless - in situ suture bridge technique in high-grade bursal side rotator cuff tears. J Orthop. 2020;20 (July):352-8.

21. Shin SJ, Jeong JH, Jeon YS, Kim RG. Preservation of bursal-sided tendon in partial-thickness articular-sided rotator cuff tears: a novel arthroscopic transtendon anatomic repair technique. Arch Orthop Trauma Surg. 2016;136(12):1701-1708. doi:10.1007/s00402-0162546-1

22. Anderson K, Aschenbrener D. Outcome of arthroscopic repair of high-grade, bursal-sided, partial rotator cuff tears (SS-04). Arthroscopy. 2004;20(1):E2-3. doi:10.1016/j.arthro.20 04.02 .005

23. Bedeir YH, Jimenez AE, Grawe BM. Recurrent tears of the rotator cuff: effect of repair technique and management options. Orthop Rev. 2018;10(7593):70-76. doi:10.4081/or.2018.7593

24. Bunker DLJ, Ilie V, Ilie V, Nicklin S. Tendon to bone healing and its implications for surgery Corresponding author: muscles. Ligaments Tendons J. 2014;4(3):343-350.
Orthopedic Research and Reviews

\section{Publish your work in this journal}

Orthopedic Research and Reviews is an international, peer-reviewed, open access journal that focusing on the patho-physiology of the musculoskeletal system, trauma, surgery and other corrective interventions to restore mobility and function. Advances in new technologies, materials, techniques and pharmacological agents are particularly welcome. The manuscript management system is completely online and includes a very quick and fair peer-review system, which is all easy to use. Visit http://www.dovepress.com/testimonials.php to read real quotes from published authors. 